\title{
Aussagekräftige Methoden zur Einschätzung des Geburtsfortschrittes
}

\author{
Franziska von Moers
}

\author{
Bachelorarbeit | Angesichts hoher Interventionsraten bei Gebärenden mit niedrigem Aus- \\ gangsrisiko ist die Entscheidungsgrundlage für Interventionen neu zu hinterfragen. Die \\ Beurteilung des Geburtsfortschrittes beeinflusst maßgeblich den Einsatz von Medikation \\ und mechanischen Interventionen. Die vaginale Untersuchung als zentraler Parameter für \\ das Voranschreiten der Geburt steht in der Fachwelt auf dem Prüfstand. Die Autorin identifi- \\ ziert mögliche Alternativen zur Beurteilung des Geburtsfortschrittes und leitet Empfehlun- \\ gen für die Geburtsbetreuung durch Hebammen ab.
}

\section{Hintergrund}

In der heutigen Geburtshilfe wird die vaginale Untersuchung als Standard zur Diagnostik des Geburtsfortschrittes verwendet [4][19][11][14]. Im klinischen Alltag bestimmen standardisierte, regelmäßige vaginale Untersuchungen die Praxis [6]. In der Regel wird in der Eröffnungsperiode alle zwei Stunden und in der Austreibungsperiode halbstündlich bzw. stündlich eine vaginale Untersuchung durchgeführt, um den kontinuierlichen Geburtsfortschritt nachzuweisen. In der Praxis werden der Literatur zufolge weitaus mehr vaginale Untersuchungen durchgeführt, als aus dem Partogramm hervorgehen [35].

Die Nice Guidelines [26] empfehlen bei einem physiologischen Geburtsverlauf eine vaginale Untersuchung alle vier Stunden, um den Geburtsfortschritt beurteilen zu können. Die WHO sowie „Evidence Based Guidelines for Midwifery-Led Care in Labour" [24] geben sogar an, dass es derzeit keine direkten Belege für die am besten geeignete Häufigkeit dieser Untersuchung gibt. Sie steht in Abhängigkeit zum Geburtskontext und Geburtsstadium [35][23][40].

Wenn Hebammen eine innere Untersuchung durchführen, gilt es zu Bedenken, dass sie damit die Konzentration der Gebärenden unterbrechen. Zum einen müssen sie um die Erlaubnis zur Untersuchung bitten. Zum anderen wird die Wehende durch die Entkleidung und Lagerung in einer günstigen Position aus dem Rhythmus des Wehen-Pausen-Musters gerissen [9]. Diese Unterbrechung kann bei der Frau Stress auf psychischer und physischer Ebene auslösen. Das möglicherweise daraus resultierende Angst-Spannungs-Schmerz-Syndrom kann das Geburtsgeschehen wesentlich verzögern und das Outcome für Mutter und Kind negativ beeinflussen [8][14].

Der Vorgang der Geburt wird durch das Gehirn gelenkt. Der Hirnstamm, der primitive Teil des Gehirns, produziert Hormone, um muttermundwirksame Kontraktionen zu erzeugen. Gleichzeitig wird der Neokortex, der emotionale Teil, auf Reserveeinheit gesetzt. Wehende Frauen ziehen sich daher im Verlaufe der Geburt zurück und wollen nicht mehr mit ihrer Umwelt in Kontakt treten, um sich auf das Geschehen in ihrem Körper zu fokussieren. Wenn der Neokortex durch externe Reize stimuliert wird, werden der Teil des Hirnstammes und die damit einhergehenden instinktiven Prozesse gehemmt. Es werden wehenhemmende Hormone wie Adrenalin, Norepinephrin und Cortisol ausgeschüttet [36]. Bewertet man die vaginale Diagnoseerhebung anhand dieser Fakten, wird klar, dass durch das gestörte hormonale Gleichgewicht eine Irritation des Hirnstammes entsteht und durch die gleichzeitige Aktivierung des Neokortex das Tempo der Geburt gedrosselt werden kann [29].

\footnotetext{
Merke

Den Vorteilen der diagnostischen vaginalen Untersuchung stehen auch verschiedene Nachteile gegenüber, die zu einer Drosselung des Geburtsfortschrittes führen können. Die vaginale Untersuchung sollte demnach nur mit streng gestellter Indikation durchgeführt werden.
} 
Die diagnostische Aussagekraft der vaginalen Untersuchung wurde zudem durch zahlreiche Forschungsteams in Frage gestellt, wobei sie eine limitierte Präzision ergab [27] [16][3][32]. Die Bewertung der vaginalen Untersuchung korreliert mit einer starken Assoziation der geburtshilflichen Erfahrung der Hebamme [4]. Das relativiert die Notwendigkeit regelmäßiger vaginaler Untersuchungen und stellt insbesondere das Untersuchen nach zeitlichen Kriterien in Frage. Eine Reihe von Studien zeigt die Limitationen und Unschärfen sowie die mangelnde Genauigkeit der Methode auf. Demnach stellt sich die Frage, inwieweit die Dominanz dieser Diagnosepraxis durch nichtinvasive Methoden ergänzt und präzisiert werden kann.

\begin{tabular}{|l|l|}
\hline \multicolumn{1}{|c|}{ Studiendesign } & \\
\hline \multirow{2}{*}{ Methode } & $\begin{array}{l}\text { Integratives Literaturreview nach Torraco, R. J. } \\
\text { (2005) [37] } \\
\text { Theoretische, empirische, qualitative und quantitative } \\
\text { Literatur beleuchtet die Thematik umfassend. } \\
\text { Die ausgewählten Studien wurden außerdem einer } \\
\text { Klassifikation nach Oxford gemäß dem „Level of Evi- } \\
\text { dence“ eingeteilt [31]. }\end{array}$ \\
\hline $\begin{array}{l}\text { Keine systematische Literaturrecherche } \\
\text { Keine thematischen empirischen Erhebungen }\end{array}$ \\
\hline \multirow{2}{*}{ Zimitation } & $\begin{array}{l}\text { Derzeitige Studienlage aufzeigen, Hinterfragen der } \\
\text { aktuellen Methoden zur Diagnostik des Geburtsfort- } \\
\text { schrittes, Implementierung der Ergebnisse in die Pra- } \\
\text { xis anregen }\end{array}$ \\
\hline $\begin{array}{l}\text { Datenbankrecherche (Pupmed, DIMDI, Cochrane Lib- } \\
\text { rary, CINAHL und Medline via OVID. } \\
\text { Vaginale Untersuchung=4 Studien } \\
\text { Wehentätigkeit=3 Studien } \\
\text { verbalen und nonverbalen Veränderungen = 1 Studie } \\
\text { Purple Line=5 Studien }\end{array}$ \\
\hline Literaturrecherche
\end{tabular}

\section{Alternative Diagnostik als Qualitätsmerkmal}

Die diagnostische Kompetenz ist ein Qualitätsmerkmal der Hebammenarbeit [10][33]. Besonders in Bezug auf den Geburtsfortschritt ist diese Fähigkeit wichtig, da sie oft über den weiteren Verlauf einer Geburt entscheidet. Es gibt unterschiedliche Methoden, um die Diagnose über das Voranschreiten der Geburt zu stellen. Hebammen sind in der Lage, diese Methoden anzuwenden, um die Frau ganzheitlich in ihrem Geburtsprozess wahrzunehmen, Anzeichen einer Veränderung im Geburtsverlauf zu erkennen und zu bewerten, um dann angemessen handeln zu können.

„Eine Hebammendiagnose ist eine Beurteilung, die von einer Hebamme [... ] nach einem Assessment (bestehend aus Beobachtung, Interview, körperlicher Untersuchung und Ressourceneinschätzung) gemacht wird. " (Georg \& Cignacco, 2006) [12]
Demnach sollten verschiedene Beobachtungen in eine aussagekräftige Einschätzung mit einfließen, um die Qualität der Aussage zu erhöhen.

Um die Physiologie der Geburt bestmöglich fördern zu können, ist es wichtig, deren Verlauf zu kennen und einschätzen zu können. Hebammen und Ärzte verlieren wichtige Fertigkeiten und Fähigkeiten, um physiologische Prozesse zu erkennen, wenn ihnen die Erfahrung mit normalen Geburtsverläufen fehlt. Zudem werden ungestörte physiologische Geburtsabläufe im klinischen Kontext immer seltener, da die Geburten durch technischen Einfluss einer Medikalisierung und einer zunehmend automatisierten Kontrolle unterliegen.

Im klinischen Setting betreuen Hebammen oft mehrere Gebärende gleichzeitig, was eine kontinuierliche Beobachtung und Konzentration auf eine Frau erschwert. Zudem wird die Beobachtung der äußerlichen körperlichen Veränderungen während der Geburt oft nicht eingesetzt oder zumindest in der Dokumentation nicht schematisch abgefragt. Aufgrund dieser Bedingungen kann der Geburtsfortschritt im Kreißsaal oft nur eingeschränkt beurteilt werden [11][20][39].

Es gibt jedoch alternative Methoden zur Beurteilung des Geburtsfortschrittes. Sie könnten als Grundlage für eine aussagekräftige Einschätzung dienen, um frühzeitige Interventionen, wie eine vaginal-operative Geburt oder einen Kaiserschnitt ggf. zu vermeiden [10].

\section{Voraussetzungen für alternative Methoden}

Um die körperlichen und emotionalen Veränderungen der Frau beobachten zu können, müsste eine Eins-zu-einsBetreuung gewährleistet sein, damit die Gebärende in ihrer Geburtsarbeit nicht gestört würde. Der Einsatz nicht invasiver Beurteilungsmethoden setzt eine kontinuierliche Betreuung der Gebärenden voraus, um Veränderungen im Geburtsprozess wahrzunehmen und palpatorische Methoden routiniert anzuwenden [15][23]. Die Beobachtung des Voranschreitens der physiologischen Geburt benötigt sowohl ein geschultes Auge als auch Zeit, um physische und psychische Anzeichen wahrzunehmen, ohne die Frau in ihrem Geburtsprozess zu stören.

\section{Alternative Methoden zur Erfassung des Geburtsfortschrittes}

Die Einteilung nicht invasiver Methoden zum Assessment des Geburtsfortschrittes kann im Wesentlichen in Beobachtende Kategorien und Haptische Kategorien klassifiziert werden. Jedoch sind einige Methoden nicht 
eindeutig einzuordnen, da ihr Anwendungsspektrum variiert werden kann. Beispielsweise können Wehen beobachtet werden in Länge und Frequenz. Die abdominale Stärke der Kontraktion bedarf allerdings einer palpatorischen Beobachtung. Im Folgenden werden drei ausgewählte Assessments vorgestellt.

\section{DEFINITION \\ Purple Line}

Wenn sich der Druck durch den kindlichen Kopf im Kreuzbein auf die Vena iliaca interna und deren Äste erhöht, staut sich das Blut an dieser Stelle und dehnt die Außenhaut der Analfurche aus. Die Spalte zwischen den Gesäßbacken wird geglättet und gibt dem Fetus mehr Raum im Becken (Hobbs in: Lewis, 2015 [22]). Mit zunehmender Zervixdilatation steigt eine violette Linie, in der Literatur als Purple Line bekannt, in der Gesäßfurche vom Analsaum kranial hoch bis zum Illiosakralgelenk [38]. Die furchenartige Ausbildung im Gesäß wird durch einen Faszienstreifen ausgebildet, der das subkutane Fettgewebe des Gesäßes minimiert. Die verlaufenden Gefäße werden mit steigender Kongestion (Zunahme der Blutmenge in bestimmten Gebieten des Körpers) vermehrt sichtbar und lassen die violette Linie zwischen den Gesäßhälften transparent durchschimmern.

\section{Purple-Line-Beobachtung}

In Bezug auf Vorkommen und Sichtbarwerden der Purple Line unterscheiden die Forscher zwischen hellen und dunklen Hauttypen. Bei hellhäutigen Frauen ist eine rosa bis rot-bräunliche Linie und bei dunkelhäutigen Frauen eine silberne Linie zu erkennen [25]. Die Autoren geben bei hellhäutigen Frauen mit einem
Prävalenzspektrum von 67,5 bis $89 \%$ das Sichtbarwerden der Purple Line an. Bei dunkelhäutigen Gebärenden werden deutlich niedrigere Zahlen angegeben [35][25][19][5]. Ebenfalls ist das Sichtbarwerden der Purple Line nach spontanem Geburtsbeginn sowie nach Blasensprung beobachtet worden [28]. Das Sichtbarwerden der Purple Line ist unabhängig von der Parität der Gebärenden.

Aufgrund der positiven Korrelation zwischen Muttermundweite und Länge der Purple Line eignet sich die Beurteilung der Purple Line als nicht-invasive Maßnahme zur Beurteilung des Geburtsfortschritts. Sheperd et al. (2011) und Narchi et al. (2011) geben beide im Streudiagramm eine mittlere positive Korrelation an [35][25]. Kordi et al. (2014) geht in seinen Ergebnissen mit einer ausgewiesenen Sensitivität von $88,9 \%$ und einer Spezifität von 48,6\% von einer Vorhersage der Purple Line von $92,3 \%$ aus [19]. Ab $7 \mathrm{~cm}$ Purple-Line-Länge gilt der Geburtsfortschritt als prognostisch günstig und $a b 10 \mathrm{~cm}$ gehen die Forscher von einem vollständig dilatierten Muttermund aus (vgl. [34])

\section{Bewertung}

Die Purple-Line-Beobachtung scheint für einen Großteil der Gebärenden anwendbar zu sein. Das Assessment wird von den Frauen als angenehm empfunden. Zudem ist die Methode auch von Hebammen mit weniger Berufserfahrung einfach praktizierbar. Die Beobachtung der Purple Line ist aufgrund der hohen Prävalenz ihres Auftretens eine gute Ergänzung zur vaginalen Untersuchung. Sie könnte sich als alternative Methode zur Einschätzung des Geburtsfortschrittes etablieren und die Aussagekraft der vaginalen Untersuchung durch die ergänzende Betrachtung präzisieren.

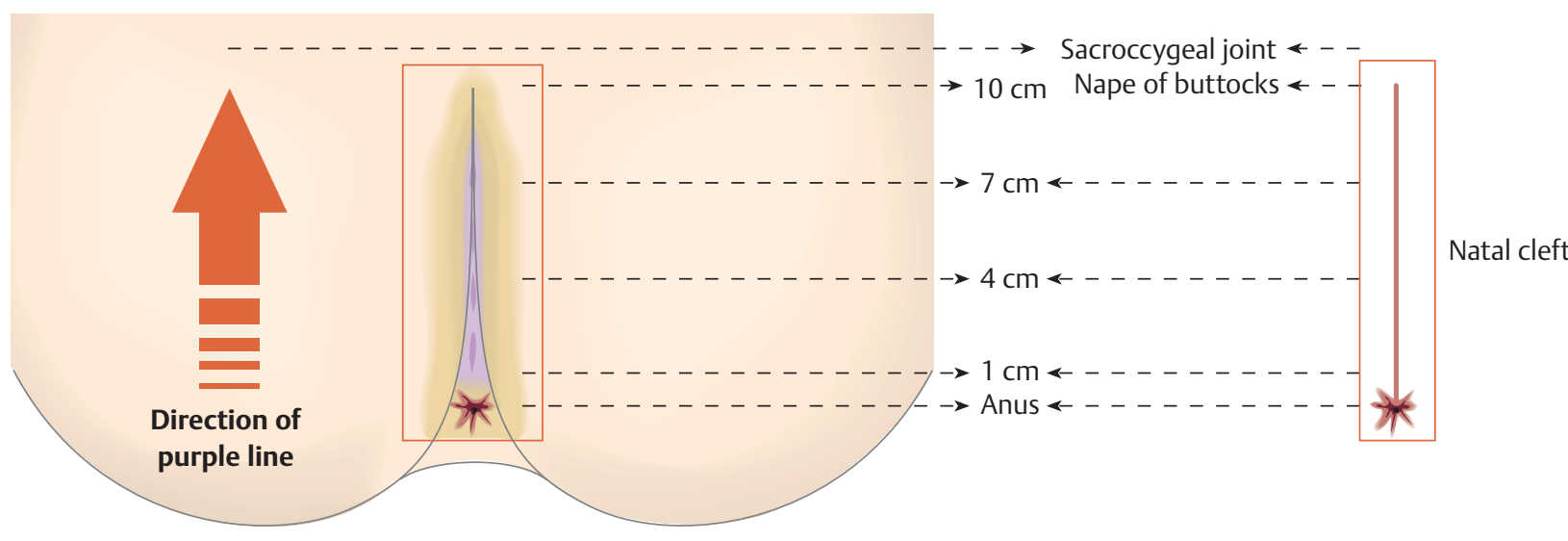

Abb. 1 Beobachtung der Purple-Line zur Einschätzung des Geburtsfortschrittes. (Quelle: nach Kordi, M, Irani M, Tara F et al. (2014) [19], graf. Umsetzung: Thieme Gruppe) 
Merke

Purple-Line-Beobachtung: Die violette Linie in der Gesäßfalte steigt während der Geburt an. Sie kann in der Intensität des Sichtbarwerdens beurteilt und zum Beispiel anhand eines Maßbandes gemessen werden.

\section{Beobachtung der Wehentätigkeit}

Die Wehentätigkeit kann sowohl beobachtet als auch palpiert werden. In einem systematischen Review von Hanley et al (2016) wird darauf verwiesen, dass $71 \%$ der 62 ausgewählten Studien den Geburtsbeginn mit regelmäßigen, schmerzhaften Wehen angeben. Die Beobachtung wurde mit den Daten des CTGs gestützt. Die Einteilung der Geburtsphasen ist außerdem abhängig von der Anzahl der Kontraktionen in einem bestimmten Zeitraum und der Länge der Wehen [14]. Demzufolge kann davon ausgegangen werden, dass Kontraktionen als Parameter zur diagnostischen Einschätzung für den Geburtsfortschritt herangezogen werden können. Sie können beobachtet und dokumentiert werden, ohne die Frau in der Geburtsarbeit zu stören.

Forscherteams verglichen in ihren Studien die Häufigkeit der Kontraktionen in der latenten und der aktiven Phase der Geburt. Als Vergleichsparameter wurde hier die vaginale Untersuchung erhoben. Die Parameter der Wehenbeobachtung bezogen sich auf Frequenz, Länge, Muster und Typus. Durch eine kontinuierliche Beobachtung der Wehen könnten demnach Veränderungen ihrer Form registriert werden und eine Tendenz über den Geburtsfortschritt ausgemacht werden. Diese Methode wird jedoch von den Forschern nur als ergänzende Einschätzung zur vaginalen Diagnostik empfohlen und dient demzufolge nur als begleitender Parameter des Geburtsfortschritts und nicht als Ersatz der vaginalen Untersuchung [14][13][41].

\section{Bewertung}

Die Beobachtung der Wehen lässt sich bei allen Gebärenden anwenden und ist leicht messbar. Diese Methode ist nicht invasiv und kann die Frequenz der vaginalen Untersuchungen ggf. reduzieren.

\section{Beobachtung von verbalen Ausdrucksformen und Gebärden}

Ausdrucksformen wie Stöhnen, Kreischen, Schreien lassen deutliche Muster für die jeweiligen Geburtsphasen erkennen und können einen Hinweis auf den Geburtsfortschritt geben. In der verblindeten, quantitativen Studie von Baker und Kenner (1993) wurde über einen Zeitraum von sechs Monaten untersucht, ob anhand von verbalen und nonverbalen Ausdrucksformen von Gebärenden die jeweilige Phase der Geburt eingeschätzt werden kann. Die Forscher verglichen mittels Videoanalyse die Stimmen der Frauen im normalen Zustand und während des Gebärprozesses. Die Analyse der aufgenommenen Stimmen zeigte im Verhältnis zur normalen Stimme eine Zunahme an Grundfrequenz und Intensität. Eine deutliche Tendenz zur Verlängerung von Lauten sowie eine Veränderung der Konturform der Grundwellenfrequenz wurden während der Geburt registriert. Die Auswertung von 40 Probanden, bestehend aus Hebammen und Ärzten, ergab einen positiven Wiedererkennungswert der Geburtsphasen von über $85 \%$ [1].

\section{Bewertung}

Es erscheint denkbar, dass die Rate an vaginalen Untersuchungen durch die Beobachtung von verbalen Ausdrucksformen und Gebärden der Frau reduziert werden könnte. Aktuellere Forschungen zu diesem Ansatz und eine genaue Definition der aussagekräftigen verbalen Veränderungen in den jeweiligen Phasen des Geburtsfortschritts wären notwendig, um diese Methode als breit anwendbares und einfach zu messendes komplementäres Assessment standardmäßig anwenden zu können.

\section{Weitere nicht invasive Methoden zur Beurteilung des Geburtsfortschrittes}

Hebammen kennen eine Vielzahl am Komplementärmethoden für die Beurteilung des Geburtsfortschrittes und wenden diese auch an. Es fehlen jedoch wissenschaftlich fundierte Grundlagen, weshalb diese Alternativassessments dem Evidenzlevel 5, dem Erfahrungswissen, zuzurechnen sind.

Beispielsweise bieten die Leopoldschen Handgriffe, die Methode nach Crichton sowie geburtshilfliche Handgriffe nach De Lee und Schwarzenbach ebenfalls Grundlagen zur Einschätzung des Verlaufes einer Geburt. Ebenfalls können „wandernde“ Herztöne, die Bauchform, sowie diverse geburtsbedingte, körperliche Veränderungen gepaart mit Bewegungsmustern und präferierten Positionen zu einer ganzheitlichen Betrachtung herangezogen werden. Indem Hebammen die qualitative Beurteilung des Geburtsfortschrittes durch quantitative Methoden und die fokussierte Beobachtung der körperlichen Veränderungen, die sich unter der Geburt vollziehen, in ihr Assessment mit einbeziehen, schaffen sie eine präzisere Grundlage, um fundierte Entscheidungen für oder gegen Interventionen zu treffen.

\section{Warum Hebammen den Geburtsfortschritt beurteilen müssen}

Die Beurteilung des Geburtsfortschrittes ist ein elementarer Bestandteil der Hebammenarbeit. Die Beobachtung von gebärenden Frauen und die Einschätzung über den Verlauf des Geburtsgeschehens gibt Hebammen die Möglichkeit, die Frau im 
jeweiligen Stadium der Geburt situationsgerecht zu unterstützen, Abweichungen von der Physiologie zu erkennen und eine sichere Atmosphäre zu schaffen [30][22]. Aus salutogenetischer Perspektive hängt demnach die angemessene Betreuung mit einer Verbesserung des Gesundheitszustands von Mutter und Kind zusammen [33].

Auch in Bezug auf das Schmerzempfinden während der Geburt hat die präzise Einschätzung des Geburtsfortschrittes einen Einfluss auf das Wohlergehen der Frau. Hebammen leiten Frauen durch den Gebärprozess und zeigen Möglichkeiten auf, wie Frauen mit dem Schmerz umgehen können [18]. Leap et al. (2010) haben in ihren Forschungsergebnissen herausgestellt, dass die kontinuierliche Geburtsbetreuung durch Hebammen ein wesentlicher Marker für eine niedrigere Rate an Interventionen ist und eine signifikant geringere Verwendung pharmakologischer Schmerzlinderung bewirkt [21]. Die Hebamme kann der Frau durch ständige Anwesenheit und direkte Rückmeldung ein erhöhtes Gefühl der Kontrolle geben und damit eine positive Schmerzbewältigung der Gebärenden fördern [30][18]. Die Frau in ihrem Selbstwertgefühl zu stärken kann den Umgang mit Geburtsschmerzen erleichtern [2], indem individuelle Bewältigungsstrategien erarbeitet werden.

\section{Merke}

Durch die Ergänzung von nicht invasiver Komplementärdiagnostik zur Einschätzung des Gebärprozesses wird die Selbstbestimmtheit der Gebärenden gefördert und die Geburt als natürliches Lebensereignis in den Vordergrund der praktizierten Geburtshilfe gestellt [7][29].

Bemerkenswert sind ebenfalls die Erkenntnisse aus der Studie von Leap et al., die eine erhöhte Ausschüttung von Oxytocin mit einer harmonischen Geburtsatmosphäre assoziieren [21]. Das bedeutet, dass Frauen, die bei der Geburt situationsangemessen betreut werden, eine bessere Aussicht auf ein physiologisches Geburtsgeschehen haben, wenn sie durch nicht invasive diagnostische Einschätzung des Prozesses keine Störung in ihrer Gebärarbeit erfahren [21]. Dieses habe auch Einfluss auf nachfolgende Geburten [30].

\section{Als forschende Hebammen Assessments weiterentwickeln}

Ein wichtiger Ansatz liegt in der Ausbildung der Hebammen [18][22]. Die International Confederation of Midwives gibt in ihrer Hebammendefinition an, dass Hebammen die physiologische Geburt fördern sollen. Daraus leitet sich ab, dass Hebammen die Anzeichen 
eines normalen Geburtsverlaufes kennen müssen, um diesen zu fördern. Hebammen sollten vorausschauend Frauen während der Geburt betreuen, ohne sie in ihrer Geburtsarbeit zu behindern [17].

Es stellt sich hier die Frage nach der Sicherheit der diagnostischen Kompetenz, in Bezug auf den Geburtsfortschritt. Wenn Hebammen ihr praktisches Repertoire durch andere Methoden erweitern sollen, ohne ihr Sicherheitsgefühl in der Befunderhebung zu verlieren, muss ihnen das neue Wissen gut vermittelt werden und sie müssen verständlich dazu angeleitet werden.

Im Zuge wissenschaftlichen Arbeitens lernen Hebammenstudierende, quantitative und qualitative Studien zu verstehen und eigene Daten zu erheben und auszuwerten. Das könnte die Hemmschwelle senken, um selbst Forschung zu betreiben. Die Akademisierung des Hebammenberufes bietet die Möglichkeit, dass werdende und forschende Hebammen verschiedene Assessments des Geburtsfortschrittes intensiver auf ihre Evidenz überprüfen, Methoden präzisieren oder ergänzen und damit die Diagnostik in den kommenden Jahren mit beeinflussen.

\section{Implementierung in die Praxis}

Eine Änderung des Partogramms zugunsten komplementärer Methoden wäre ein wünschenswerter Ansatz, um die Arbeit und die Wahrnehmung des Hebammenberufes zu verändern. Die Implementierung dieser Methoden durch routinierte Abfrage in der Dokumentation, beispielsweise im klinischen Kontext, würde den Beruf der Hebamme durch eine vielseitige Beurteilung des Geburtsfortschrittes in der Kompetenz und dem fachlichen Können qualitativ aufwerten. Ebenfalls würde durch eine bessere Diagnostik, eine gesteigerte Qualität der Betreuung und eine höhere Expertise das Hebammenhandwerk mehr Beachtung finden und den Berufsstand in seiner Einzigartigkeit qualitativ anheben. Damit es dazu kommen kann, müssen Ausbildung und Forschung viel stärker verwoben werden, als es bisher der Fall ist. Forschung und Entwicklung müssen zum Gegenstand staatlicher Forschung werden.

\section{Autorinnen / Autoren}

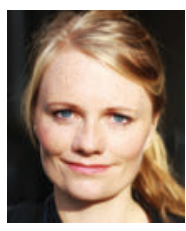

Franziska von Moers ist Hebamme B.Sc. und arbeitet als Kreißsaalhebamme im Sana Klinikum in Berlin. Ihre hier zusammengefasste Bachelorarbeit an der Evangelischen Hochschule Berlin verfasste sie zum Thema: Assessment des Geburtsfortschrittes. Welche Methoden tragen zu einer aussagefähigen Einschätzung des Geburtsfortschrittes bei?
Korrespondenzadresse

E-Mail: fvonmoers@gmail.com

Literatur

[1] Baker A. \& Kenner A. N. (1993). Communication of Pain: Vocalization as an Indicator of the Stage of Labour. australian new zealand journal obstetrics gynaecology, 33: 4: 384.

[2] Berentson-Shaw J, Scott KM, Jose PE. (2009). Do sel-efficacy beliefs predict the pri-miparous labour and birth experience? A longitudinal study. J Reprod Infant Psychol; 27: 357-73

[3] Buchmann E. J. \& Libhaber E. (2007). Accuracy of cervical assessment in the active phase of labour. BJOG International Journal of Obstretics and Gynoaecology, 144:833-837. doi: 10.1111/j.1471-0528.2007.01386

[4] Brancadoro M.; Tognarelli S.; Fambrini F.; Ragusa A.; Menciassi A. (2018). Devices for Measuring Cervical Dilation During Labor: Systematic Review and Meta-analysis. Obtretrical and Gynecological survey, doi: 10.1097/OGX.0000000000000548.

[5] Bryne D. L. \& Edmonds D. K. (1990). Clinical method for evaluating progress in first stage of labour. The Lancet, 335:122.

[6] Chalubinski K. M. (2016). Normale Geburt. In: Schneider H., Husslein P., Schneider K. T. M. (Hrsg.), Die Geburtshilfe (S. 663-6

[7] Davies L. (2011). Sitting next to Nellie: Vaginal examinations in normal labour. Essen-tially MIDIRS 2 (4),S. 38-42

[8] Dick-Read G. (2006). Childbirth without Fear. London: Pollinger.86). (2. Aufl.). Berlin: Springer

[9] Dixon L, Skinner J, Foureur M. (2014). The emotional journey of labourwomen's per-spectives of the experience of labour moving towards birth. Midwifery 2014;30:371-7.

[10] Deutsches Netzwerk für Qualitätsentwicklung in der Pflege (DNQP) \& Verbund Heb-ammenforschung (Hrsg.) (2015). Expertinnenstandard Förderung der physiologischen Geburt. Osnabrück: Schriftenreihe des DNQP.

[11] Downe S.; Gyte GM.; Dahlen HG; Singata M. (2013). Routine vaginal examinations for assessing progress of labour to improve outcomes for women and babies at term., doi: 10.1002/14651858.CD010088.pub2., Cochraine Database systematic review

[12] Georg J., \& Cignacco E. (2006). Hebammendiagnosen und deren Bedeutung für die Professionalisierung des Hebammenberufes. In: Cignacco E.(Hrsg.), Hebammenarbeit (S. 255-281). Bern: Huber.

[13] Hamilton E. F., Warrick P. A., Collins K., Smith S., \& Garite T. J. (2016). Assessing first-stage labor progression and its relationship to complications. American Journal of Obstetrics and Gynecology, 214(3), 358. https://doi.org/10.1016/j. ajog.2015.10.016

[14] Hanley G. E., Munro S., Greyson D., Gross M. M., Hundley V., Spiby H., \& Janssen P. A. (2016). Diagnosing onset of labor: a systematic review of definitions in the research literature. BMC Pregnancy and Childbirth, 16 (03). https://doi.org/ 10.1186/s12884-016-0857-4

[15] Hodnett E. D., Gates S.; Hofmeyr G. J.; Sakala C.; Weston J. (2011): Continous support for women during childbirth (Review): The Cochrane Collaboration. The Cochrane Library 2.

[16] Huhn K. A. \& Brost B. (2004). Accuracy of simulated cervical dilatation and efface-ment measurements among 
practitioners. American Journal of Obstetrics and Gynecology 191: 1797-1799.

[17] International Confederation of Midwives (ICM). (2017) International definition of the midwife. Aufgerufen unter: https: / / www.internationalmidwives.org/our-work/policy-and-prac tice/icm-definitions.html (letzter Zugriff am 11.03.2019)

[18] Karlsdottir S.; Sveinsdottir H.; Kristijansdottir H.; Aspelund T.; Olafsdottir O. (2017). Predictors of women's positive childbirth pain experience: Findings from an Icelandic national, Women and Birth 31 (2018) e178-e184, Elsevier

[19] Kordi M.; Irani M.; Tara F. \& Esmaily H. (2014). The Diagnostic Accuracy of Purple Line in Prediction of Labor Progress in Omolbanin Hospital, Iran. Iranian Red Cresent Medical Journal, 16 (11): e16183. doi:10.5812/ircmj. 16183

[20] Lavender T.; Hart A.; Smyth RM. (2013). Effect of partogram use on outcomes for women in spontaneous labour at term, the Cochrane database of systematic reviews doi: 10.1002/ 14651858.CD005461.pub4.

[21] Leap N.; Sandall J.; Buckland S.; Huber U. (2010). Journey to confidence: women's experiences of pain in labour and relational continuity of care, Journal of Midwifery and women's health, American College of Nurse-Midwives; Elsevier, doi: 10.1016/j.jmwh.2010.02.001.

[22] Lewis L. (2015). Fundamentals of Midwifery: A Textbook for Students. Chichester: Wiley.

[23] Lindsay P., Bagness C., \& Peate I. (2018). Midwifery Skills at a Glance. Oxford: Wiley.

[24] Munro J. \& Jokinen M. (2012). Evidence Based Guidelines for Midwifery-Led Care in Labour. Assessing Progress in Labour. The Royal College of Midwives Trust. Retrieved from http: / / www.rcm.org.uk/college/policy-practice/evidence-basedgui delines/(Abgerufen am 13.06.2019)

[25] Narchi N. Z., Camargo J. C. S., Salim N. R.; Menezes M. O. \& Bertolino M. M. (2011). Utilização da "linha púrpura" como método clínico auxiliar para avaliação da fase ativa do trabalho de parto. Revista Brasileira de Saúde Materno Infantil, 11 (3), 313-322. https://doi.org/10.1590/s151938292011000300012

[26] National Institute for Health and Care Excellence (NICE) (2014). Intrapartum care: care of healthy women and their babies during childbirth, clinical guideline 190 .

[27] Nizard J.; Haberman S.; Paltieli Y.; Gonen R.; Ohel G.; Nicholson D.; Ville Y. (2009). How reliable is the determination of cervical dilation? Comparison of vaginal examination with spatial position-tracking ruler. American Journal of obstretrics and gynecology, doi: 10.1016/j.ajog.2009.01.002.

[28] Nunes R. D.; Locatelli P.; Traebert J. (2018)Use of the purple line to diagnose cervical dilatation and fetal head station during labor, May;141(2):250-251. doi: 10.1002/ijgo.12459.

[29] Odent M. (1992): Geburt und Stillen. Über die Natur elementarer Erfahrungen. Mün-chen. Beck
[30] Olza I.; Leahy-Warren P.; Benvamini Y.; Kazmierczak M.; Karlsdottir S.; Spyridou A. et al. (2018). Women's psychological experiences of physiological childbirth: a meta-synthesis, bjm open, doi:10.1136/bmjopen-2017-020347

[31] Oxford Centre for Evidence-based Medicine - Levels of Evidence (2009) - CEBM. (auf-gerufen unter: https: / /www.ceb m.net/2009/06/oxford-centre-evidence-based-medicine-lev els-evidence-march-2009/, letzter Zugriff am 30.06.2019)

[32] Phelps J. Y., Higby K., Smyth M. H., Ward J. A., Arredondo F., Mayer A. R. (1995). Accuracy and intraobserver variability of simulated cervical dilatation measurements. American Journal of Obstetrics and Gynecology, 173, 942-945.

[33] Renfrew M. J., McFadden A., Bastos M. H., Campbell J., Channon A. A., Cheung Audebert Delage Silva D. R.; Downe S.; Kennedy H. P.; Malata A.; McCormick F., Wick L.; Declercq E.; McCormick F. (2014). Midwifery and quality care: findings from a new evidence-informed framework for maternal and newborn care, The Lancet, 384 (9948), 1129-1145 http: / dx. doi.org/10.1016/S0140-6736(14)60789-3

[34] Sheperd A., Cheyne H., Kennedy S., Mclntosh C., Styles M. \& Niven C. (2010). The purple line as a measure of labour progress: a longitudal study. BMC Pregmancy and Childbirth. 10:54-60. doi:10.1186/1471-2393-10-54

[35] Sheperd A. \& Cheyne H. (2011). The frequency and reasons for vaginal examinations in labour. doi:10.1016/j. wombi.2012.02.001

[36] Simkin P. \& Klaus P. (2015). Wenn missbrauchte Frauen Mütter werden. Stuttgart: Klett-Cotta

[37] Torraco R. J. (2005). Writing Integrative Literature Reviews: Guidelines and Examples. Human Resource Development Review 4 (3): 356-367.

[38] Walsh D. (2007). Evidence-based Care for Normal Labour and Birth: A guide for mid-wives. New York: Taylor \& Francis Group.

[39] Walsh D. (2010a): Labour rhythms vs labour progress: one final update. British Journal of Midwifery, 18 (8), S.482

[40] World Health Organization (2018). WHO recommendations Intrapartum care for a posi-tive childbirth experience, Geneva: Licence: CCBY-NC-SA3.0IGO

[41] Zagami S.E. MSC, Golmakani N. MSC, Ali-Reza Saadatjoo S. MSC, Ghomian N. MD \& Baghbani B. BSC. (2013). The Shape of Uterine Contractions and Labor Pro-gress in the Spontaneous Active Labor. Iranian journal of medical sciences, 40 (02). Ab-gerufen von https://www.ncbi.nlm.nih.gov/pm c/articles/PMC4359943/

\section{Bibliografie}

DOI https://doi.org/10.1055/a-1084-5255

Die Hebamme 2020; 33: 59-65

(c) Georg Thieme Verlag KG Stuttgart · New York ISSN 0932-8122 